\title{
Green tea polyphenols increase p53 transcriptional activity and acetylation by suppressing class I histone deacetylases
}

\author{
VIJAY S. THAKUR ${ }^{1,2}$, KARISHMA GUPTA ${ }^{1}$ and SANJAY GUPTA ${ }^{1-3}$ \\ ${ }^{1}$ Department of Urology and Nutrition, Case Western Reserve University; ${ }^{2}$ Department of Urology, University \\ Hospitals Case Medical Center; ${ }^{3}$ Case Comprehensive Cancer Center, Cleveland, OH 44106, USA
}

Received December 22, 2011; Accepted February 1, 2012

DOI: $10.3892 /$ ijo.2012.1449

\begin{abstract}
Acetylation of the tumor suppressor gene p53 at the carboxy-terminal lysine (Lys) residues enhances its transcriptional activity associated with cell cycle arrest and apoptosis. Histone deacetylases (HDACs), a family of evolutionarily conserved enzymes, counterbalance the acetylation of lysine residues on histone and non-histone proteins. In this study, we demonstrate that green tea polyphenols (GTPs) and their major constituent, (-) epigallocatechin-3-gallate (EGCG), activate p53 through acetylation at the Lys373 and Lys382 residues by inhibiting class I HDACs in LNCaP human prostate cancer cells. Treatment of cells with GTPs $(2.5-10 \mu \mathrm{g} / \mathrm{ml})$ and EGCG (5-20 $\mu \mathrm{M})$ resulted in dose- and time-dependent inhibition of class I HDACs (HDAC1, 2, 3 and 8), albeit at varying levels. Discontinuation of treatment with GTP/EGCG resulted in the loss of p53 acetylation at both the sites in these cells. GTP/ EGCG treatment also resulted in increased expression of p21/waf1 and Bax at the protein and message levels in these cells. The increased GTP/EGCG-mediated p53 acetylation enhanced its binding on the promoters of p21/wafl and Bax, which was associated with increased accumulation of cells in the G0/G1 phase of the cell cycle and induction of apoptosis. Our findings indicate that GTP/EGCG causes acetylation of p53 by inhibiting class I HDACs, a function that is likely to be part of the mechanisms that control the physiological activity of $\mathrm{p} 53$.
\end{abstract}

Correspondence to: Dr Sanjay Gupta, Department of Urology and Nutrition, Case Western Reserve University, 10900 Euclid Avenue, Cleveland, $\mathrm{OH}$ 44106, USA

E-mail: sanjay.gupta@case.edu

Abbreviations: EGCG, epigallocatechin-3-gallate; TSA, trichostatin A; GTPs, green tea polyphenols; HDACs, histone deacetylases; SAHA, suberoylanilide hydroxamic acid

Key words: prostate cancer, green tea polyphenols, epigallocatechin-3-gallate, p53 acetylation, histone deacetylases

\section{Introduction}

The tumor suppressor protein, p53, plays a critical role in inducing cell cycle arrest, DNA repair and apoptosis in response to various external and internal stresses, and protects cells from undergoing malignant transformation. In the vast majority of cancers, the p53 gene is mutated, resulting in the loss of p53 function in the central DNA binding domain (amino acid 102-292), an event that is thought to contribute to the development of malignancy. Functional domains within p53 are the $\mathrm{N}$-terminal domain, which harbors transactivation activity (amino acids 1-42), a C-terminal region that includes the tetramerization domain (amino acid 324-355) and a regulatory domain (amino acid 363-393). In a number of cancers, although wild-type p53 protein may be present, its functional response is lost, either due to post-translational modifications of the extreme C-terminal 30 residues that play important roles in the regulation of p53-specific DNA binding activity, and/or due to defects in other genes in the p53 response pathway. In normal cells, p53 is maintained at a very low level due to continuous ubiquitination by murine double minute 2 (MDM2), whereas during cellular stress p53 is phosphorylated and acetylated and, in turn, plays an important role in the stabilization and activation of p53. For example, p53 causes cell cycle arrest primarily by activating the transcription of cyclin-dependent kinase (CDK) inhibitor p21/waf1 and induces apoptosis by the transcriptional activation of the pro-apoptotic Bcl2 family genes, Bax, PUMA and Noxa $(1,2)$.

Previous studies have demonstrated that p300/CBP, the histone acetyltransferase, strongly potentiates p53-dependent transcriptional activation, and that acetylation of p53 by p300 markedly stimulates its sequence-specific DNA binding and transcriptional activity. The synergistic enhancement of p53 transactivation activity is a result of at least two different pathways, including acetylation of the core histone proteins and p53 acetylation. Post-translational acetylation of p53 at its C-terminal domain has previously been demonstrated in both in vitro and in vivo studies (2). Lysine (Lys)373 and Lys382 are acetylated by p300/CBP, and Lys320 is acetylated by the p300/ CBP-associated factor (PCAF) (2). Acetylation of p53 markedly enhances its sequences specific DNA binding activity and is induced in response to DNA damage. Besides p53, numerous 
other non-histone transcription factors have been shown to be acetylated by histone acetyltransferases (2).

Histone acetylation is a reversible process and acts in concert with histone deacetylases (HDACs) a family of evolutionary conserved enzymes that modulate the acetylation status of histones and a number of other regulatory and structural proteins. HDACs are active components of transcriptional corepressor complexes. HDACs are broadly classified into four classes based on their sequence homology, as follows: class I (HDACs1-3 and 8), class II (HDACs 4-7 and HDACs 9-10), class III (Sirt1-Sirt7) and class IV (HDAC11). Class I HDACs contain a deacetylase domain and are the homologs of yeast RPD3, whereas class II HDACs are homologs of yeast Hda1. Class III (Sirt1-Sirt7) HDACs are homologs of yeast silent mating type information regulation 2 (Sir2) and form a structurally distinct class of NAD-dependent enzymes, and class IV HDACs (HDAC11) have properties of both class I and class II HDACs (3). These proteins are localized in both the cytoplasm and nucleus, suggesting that they have diverse cytoplasmic and nuclear substrates, and are frequently overexpressed in a number of human cancers. Their differential expression often correlates with drug resistance and poor prognosis, which makes them attractive targets in cancer therapeutics (4). HDAC inhibitors have shown considerable promise as therapeutic agents for the treatment of human cancer and a number of other diseases. HDAC inhibitors such as sodium butyrate, trichostatin A (TSA), suberoylanilide hydroxamic acid (SAHA), valproic acid and trapoxins have divergent structures and promote growth arrest, differentiation, and apoptosis of tumor cells (5). Studies have shown that the proportion of acetylated p53 increases when cells are treated with HDAC inhibitors, such as TSA. This increasing level of p53 acetylation with HDAC inhibitors prevents p53 degradation thereby maintaining a tight regulation of functional p53 levels by acetylation and deacetylation $(6,7)$.

In search of safe and effective HDAC inhibitors, in our previous studies we demonstrated that green tea polyphenols (GTPs) and their major constituent, (-) epigallocatechin-3-gallate (EGCG), have the ability to inhibit class I HDACs in human prostate cancer cells. Our studies further demonstrate that GTPs enhance proteasomal degradation of class I HDACs and increase acetylation of core histone proteins, promoting access to the promoter region of CDK inhibitor (p21/waf1) and pro-apoptotic gene (Bax) in human prostate cancer cells, and stimulating cell cycle arrest and apoptosis (8). In this study we demonstrate, for the first time, that GTPs and their major constituent, (-) EGCG, increase the acetylation of p53 at Lys373 and Lys382, but not in Lys320 of its C-terminus, as a consequence of class I HDACs inhibition. The enhanced binding of acetylated p53 in the p21/wafl and Bax gene promoters increases their mRNA and protein levels, which may in part be accountable for increased cell cycle arrest and apoptosis of human prostate cancer LNCaP cells harboring wild-type p53.

\section{Materials and methods}

Cell culture and reagents. Human prostate cancer $\mathrm{LNCaP}$ cells were obtained from the American Type Culture Collection (Manassas, VA, USA). Cells were grown and maintained in RPMI-1640 (Hyclone) supplemented with $1 \%$ penicillin-streptomycin and $10 \%$ fetal bovine serum at $50-70 \%$ confluency. Cells received the following treatments: $20 \mathrm{ng} / \mathrm{ml}$ TSA (Sigma, St Louis, MO), dissolved in DMSO; 2.5-10 $\mu \mathrm{g} / \mathrm{ml}$ polyphenon $\mathrm{E}^{\circledR}$ (Mitsui Norin, Tokio, Japan) hereafter referred to as GTP; 5-20 $\mu \mathrm{M}$ EGCG (Mitsui Norin) for indicated times. Concentrations of $10 \mu \mathrm{g} / \mathrm{ml}$ polyphenon $\mathrm{E}$ correspond to $14 \mu \mathrm{M}$ EGCG. The constituents present in Polyphenon $\mathrm{E}^{\circledR}$ are mentioned in our previous publication (9).

Western blot analysis. Cells were lysed in radioimmunoprecipitation assay (RIPA) buffer (1\% NP40, 0.5\% sodium deoxycholate, $0.1 \%$ SDS in PBS containing complete protease inhibitor cocktail (Roche Applied Sciences, Mannheim, Germany). Protein concentration in the cell lysates was determined using detergent-compatible protein assay from Bio-Rad (Hercules, CA). Protein samples were subjected to SDS-PAGE and transferred to a nitrocellulose membrane. The membrane was incubated with primary antibody overnight after blocking in 5\% non-fat milk in TBS for $1 \mathrm{~h}$. The following day, the membrane was removed from the primary antibody, washed with washing buffer and subsequently incubated with appropriate horseradish peroxidase (HRP)-conjugated secondary antibody for $1 \mathrm{~h}$. The membrane was than developed with enhanced chemiluminescence reagents (GE Healthcare, Piscataway, NJ) and exposure to Hyblot CL autoradiography film (Denville Scientific, Metuchen, NJ). Image digitization and quantification were performed with the Kodak 2000 imaging system. Acetyl p53 (Lys373) was obtained from Millipore (Billerica, MA) (Cat no. 06-916), Ac-p53 (Lys382) was purchased from Epitomics (Burlingame, CA) (Cat\#2485-1), Ac-p53 (Lys320) was procured from Millipore (Cat no. 06-915) and Anti-p53 (SC-126), anti-p21/waf1 (SC-397), anti-Bax (SC-) anti-HDAC1 (SC-7872), anti-HDAC2 (SC-6296), anti-HDAC3 (SC-11417), anti-HDAC8 (SC-11405) and anti- $\beta$-actin (SC-47778) antibodies were purchased from Santa Cruz Biotechnology (Santa Cruz, CA).

Reverse-transcriptase PCR. Total RNA was extracted from various controls and treated human prostate cancer LNCaP cells using TRIzol reagent and the first-strand cDNA was prepared from $2 \mu \mathrm{g}$ total RNA using a high capacity cDNA Reverse Transcription kit (Applied Biosystems). Levels of p21/ waf1 and Bax mRNA were quantified by PCR and normalized to glyceraldehyde-3-phosphate dehydrogenase (GAPDH). The PCR reaction conditions used were: stage $1,94^{\circ} \mathrm{C}$ for $5 \mathrm{~min}$ (1 cycle); stage $2,94^{\circ} \mathrm{C}$ for $1 \mathrm{~min}$ and $58^{\circ} \mathrm{C}$ for $45 \mathrm{sec}$ and $72^{\circ} \mathrm{C}$ for $1 \mathrm{~min}$ (30 cycles); and stage $3,72^{\circ} \mathrm{C}$ for $7 \mathrm{~min}$ (1 cycle) using the ABI Veriti thermal cycler. The primers used were: p21 forward, 5'-ATTAGCAGCGGAACAAGGAGTCAG ACAT-3' and reverse, 5'-CTGTGAAAGACACAGAACAGT ACAGGGT-3'; Bax forward, 5'-GGCCCACCAGCTCTGA GCAGA-3' and reverse, 5'-GCCACGTGGGCGTCCCAA AGT-3'; and GAPDH forward, 5'-CAACGGATTTGGTC GTATTGG-3' and reverse, 5'-GCAACAATATCCACTTT ACCAGAGTTAA-3'.

Chromatin immunoprecipitation (ChIP) assay. LNCaP human prostate cancer cells were treated with various doses of GTPs or EGCG dissolved in PBS or only with PBS (control) for 3 days. At the end of treatment, cells were incubated in media 
containing $1 \%$ formaldehyde for $15 \mathrm{~min}$ at room temperature for cross-linking. The reaction was then terminated using a $0.125 \mathrm{M}$ final concentration of glycine. After cross-linking, cells were lysed, chromatin was digested using monococcal nuclease enzyme and incubated with anti-acetylated (Lys373) (Cat no. 06-916; Millipore) antibody overnight at $4^{\circ} \mathrm{C}$. After reversing the cross-linking by incubating the samples at $65^{\circ} \mathrm{C}$ overnight, DNA was purified using phenol-chloroform-isoamyl reagent followed by ethanol precipitation. DNA was then dissolved in nuclease-free water. Immunoprecipitated DNAs, beads or input controls were subjected to PCR amplification for 30 cycles of the following cycling conditions: stage $1,95^{\circ} \mathrm{C}$ for $2 \mathrm{~min}(1 \mathrm{cycle})$; stage $2,95^{\circ} \mathrm{C}$ for $30 \mathrm{sec}$ and $60^{\circ} \mathrm{C}$ for $30 \mathrm{sec}$ and $72^{\circ} \mathrm{C}$ for $1 \mathrm{~min}$ (30 cycles); and stage $3,72^{\circ} \mathrm{C}$ for $3 \mathrm{~min}$ (1 cycle). Primers used were as follows: p21/waf1 gene promoter forward, 5'-GTGGCTCTGATTGGCTTTCTG-3' and reverse, 5'-GTGAAAACAGGCAGCCCAAG-3'l and Bax gene promoter forward, 5'-TAATCCCAGCGCTTTGGAA-3' and reverse, 5'-TGCAGAGACCTGGATCTAGCAA-3'. PCR products were subjected to electrophoresis using a $2 \%$ agarose gel.

Cell cycle analysis. The effect of GTPs and EGCG on the cell cycle was measured by performing flow cytometric assay. LNCaP cells were treated with GTPs or EGCG and were harvested by trypsinization on days 3 and 7 post-treatment. Cells were washed twice with PBS. Approximately $1 \times 10^{6}$ cells were fixed in $90 \%$ cold methanol and left on ice for at least $30 \mathrm{~min}$ and stored at $-20^{\circ} \mathrm{C}$ until being processed for cell cycle. Cells were washed, pelleted and resuspended in $0.04 \mu \mathrm{g} / \mathrm{ml}$ propidium iodide and $100 \mu \mathrm{g} / \mathrm{ml}$ RNase in PBS. The samples were incubated at room temperature for $30 \mathrm{~min}$ and flow cytometry was performed on the EPICS-XL MCL flow cytometer and analyzed using Cell Quest Analysis software Modifit to determine the number of cells in each phase of the cell cycle.

Detection of apoptosis. To determine apoptosis in the control and treated LNCaP cells, cells in the sub-G1 phase were determined by flow cytometery. The assay was performed as mentioned above in the cell cycle analysis except that floaters were also collected along with live cells. Cells in the sub-G1 phase were determined using Cell Quest Analysis software Modifit.

Statistical analysis. One-way analysis of variance (ANOVA) was performed to assess the differences between groups. Differences in means among treatments were evaluated by the Dunnett's test, and the level of significance was designated as follows: ${ }^{*} \mathrm{P}<0.05,{ }^{* *} \mathrm{P}<0.001$.

\section{Results}

GTPs and EGCG decrease protein expression of class I HDACs in human prostate cancer cells. We have previously shown that low doses of GTPs mimicking physiologically attainable doses inhibit class I HDACs in human prostate cancer cells in a timedependent manner (9). We have also demonstrated that GTP treatment at higher doses $(10-80 \mu \mathrm{g} / \mathrm{ml})$ for $24 \mathrm{~h}$ decreases levels and activity of class I HDACs in a dose- and time-dependent manner partly by increasing their proteasomal degradation (8). In this study, we provide additional confirmation that exposure of LNCaP human prostate cancer cells to GTPs and their major constituent, EGCG, significantly decreases the protein expression of class I HDACs in both a dose- and time-dependent fashion. We performed western blot analysis on total cell lysates of LNCaP cells treated with various concentrations of GTPs or EGCG for 3 and 7 days. Cells treated with $20 \mathrm{ng} /$ $\mathrm{ml}$ TSA, were used as the positive controls. Treatment with GTPs as well as EGCG affected the levels of class I HDACs; however, the extent of the effect was not similar on all class I HDACs. Compared to the controls, the treatment of cells with $2.5-10 \mu \mathrm{g} / \mathrm{ml}$ doses of GTPs caused a decrease of $8-10 \%$ in HDAC1, 10-47\% in HDAC2, 8-40\% in HDAC3 and $10-12 \%$ in HDAC8 levels after 3 days; and a marked decrease of $22-60 \%$ in HDAC1, 42-74\% in HDAC2, 41-58\% in HDAC3 and 10-22\% in HDAC8 levels after 7 days of treatment. Similarly, EGCG treatment at the doses of 5-20 $\mu \mathrm{M}$ caused a decrease of $6-9 \%$ in HDAC1, 3-19\% in HDAC2, 19-40\% in HDAC3 and 2-12\% in HDAC8 levels after 3 days; and a significant decrease of $12-48 \%$ in HDAC1, 30-74\% in HDAC2, 38-56\% in HDAC3 and $19-50 \%$ in HDAC8 levels after 7 days of treatment. TSA exposure, however, caused a modest decrease of $4 \%$ in HDAC3, $8 \%$ in HDAC 8 and marked decrease of $18 \%$ in HDAC2 levels, whereas no changes were observed in HDAC1 protein levels after 2 days of treatment (Fig. 1A and B).

GTPs and EGCG promote increased stabilization and acetylation of p53 at Lys373 and Lys382 in human prostate cancer cells. We then evaluated whether changes in the homeostasis of class I HDACs after GTP or EGCG treatment in cancer cells leads to post-translational modification in the p53 tumor suppressor. For these experiments, LNCaP cells were treated with similar doses of GTPs and EGCG for the same aounts of time as described in the previous experiment. Treatment of cells with 2.5-10 $\mu \mathrm{g} / \mathrm{ml}$ GTPs resulted in a significant increase in the levels of total p53 (102-160\%) after 3 days of treatment, as well as after 7 days of treatment (120-188\%), compared to the endogenous p53 levels in untreated cells. Similar responses were noted with EGCG exposure, which caused an increase of $131-155 \%$ after 3 days and $145-198 \%$ after 7 days in total p53 at the doses of 5-20 $\mu \mathrm{M}$, respectively. The levels of acetylated p53 at Lys373 and Lys382 were significantly enhanced by exposure to both GTPs and EGCG. Compared to the controls, GTP treatment caused an increase of $112-131 \%$ in the acetylation of Lys 373 and 120-155\% of Lys382 after 3 days, and 101-133\% of Lys373 and 123-200\% of Lys382 after 7 days of exposure. Similarly, with EGCG exposure, we noted an increase of $116-124 \%$ acetylation of Lys373 and 122-133\% of Lys382 after 3 days, increasing to $120-181 \%$ of Lys 373 and $125-185 \%$ of Lys382 after 7 days of exposure, indicating that both GTPs and EGCG increase acetylation of p53 on its C-terminal by modulating class I HDACs. No changes were observed in p53 acetylation of Lys320 residue by GTPs or EGCG (data not shown). TSA exposure, however, resulted in increased acetylation (110\% in Lys373 and 149\% in Lys382 in p53), whereas no changes were observed in total p53 protein levels after 2 days of treatment (Fig. 2A and B). Our results are in agreement with previously published studies demonstrating that GTPs and EGCG upregulate p53 levels and activity. However, to the best of our knowledge, there are no reports demonstrating the effects of GTPs and/or EGCG on p53 acetylation. 
A

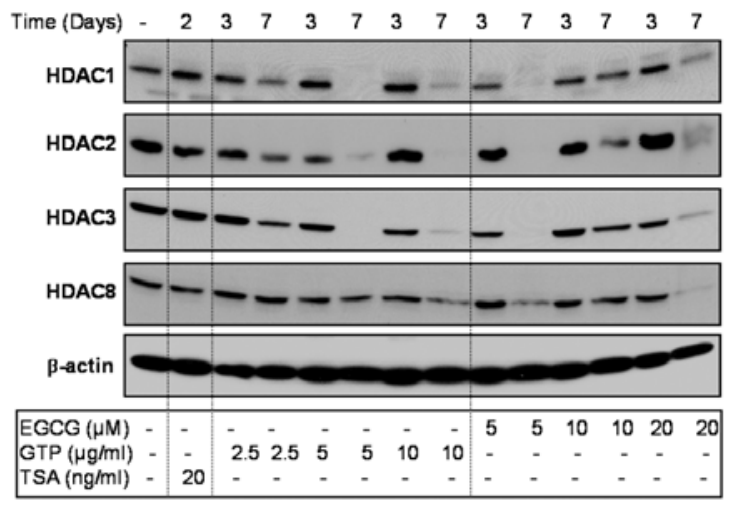

B

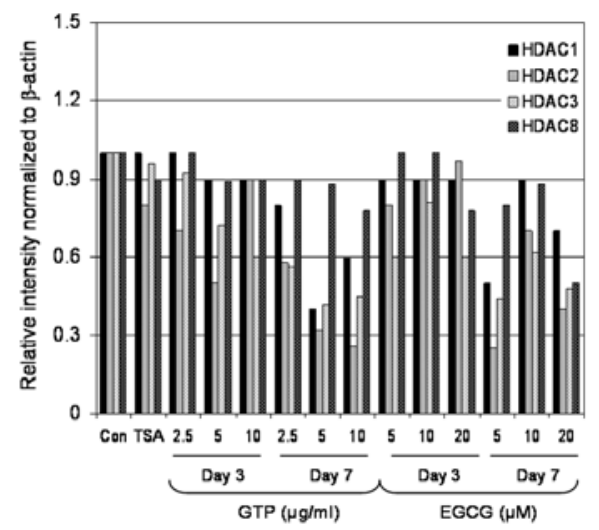

Figure 1. Effect of green tea polyphenols (GTPs) and their major constituent (-) epigallocatechin-3-gallate (EGCG) and the HDAC inhibitor, trichostatin A (TSA), on class I HDAC protein expression in LNCaP human prostate cancer cells. (A) Time-dependent inhibition of class I HDAC proteins at indicated doses of treatment with GTPs $(2.5-10 \mu \mathrm{g} / \mathrm{ml})$, EGCG $(5-20 \mu \mathrm{M})$ and the HDAC inhibitor, TSA (20 $\mathrm{ng} / \mathrm{ml})$, in LNCaP human prostate cancer cells. (B) Relative intensities of HDAC protein bands normalized to $\beta$-actin in LNCaP cells. The details are provided in Materials and methods.

A

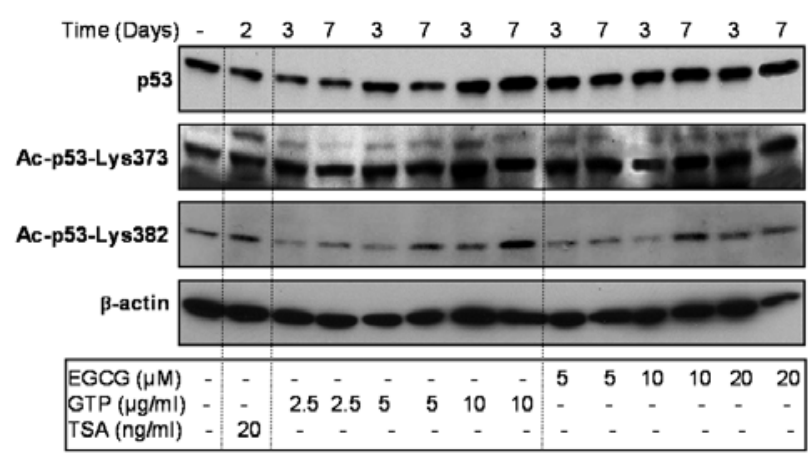

B

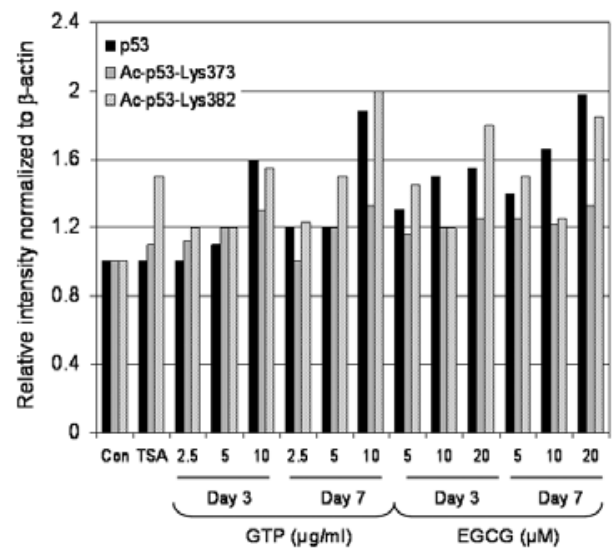

Figure 2. Effect of green tea polyphenols (GTPs) and their major constituent (-) epigallocatechin-3-gallate (EGCG) and the HDAC inhibitor, trichostatin A (TSA), on p53 protein levels and its acetylation on C-terminal Lys373 and Lys382 in LNCaP human prostate cancer cells. (A) Cells were treated with GTPs $(2.5-10 \mu \mathrm{g} / \mathrm{ml})$ or EGCG $(5-20 \mu \mathrm{M})$ for 3 and 7 days or TSA $(20 \mathrm{ng} / \mathrm{ml})$ for 2 days and western blotting was performed for p53, Ac-p53-Lys373 and Ac-p53-Lys382 proteins. (B) Relative intensities of p53 protein and its acetylation on C-terminal Lys373 and Lys382 bands normalized to $\beta$-actin in LNCaP cells. The details are provided in Materials and methods.

Acetylation of p53 induced by GTPs and EGCG is reversible in human prostate cancer cells. To further investigate whether GTP- and EGCG-induced p53 acetylation is reversible, we treated LNCaP human prostate cancer cells with either $5 \mu \mathrm{g} / \mathrm{ml}$ of GTPs or $20 \mu \mathrm{M}$ of EGCG. Cells were harvested for western blot analysis after $0,1,3,5$ and 7 days of exposure. After 7 days of exposure, the cells from some plates were replenished with culture medium without GTPs or EGCG for an additional 7 days, and then harvested for protein analysis. Compared to the untreated controls, a progressive increase in total p53 protein levels and progressive acetylation at Lys373 and Lys382 were observed up to 7 days of treatment. Increases of $161 \%$ in total p53, 180\% in Lys373 and 156\% in Lys382 were observed after 7 days of GTP treatment. In contrast, reductions of $51 \%$ in total p53, $99 \%$ in Lys373 and 46\% in Lys382 were noted after 7 days of GTP withdrawal. Similar results were noted with EGCG exposure: increases of $155 \%$ in total p53, 112\% in Lys373 and $149 \%$ in Lys382 were observed after 7 days, compared to the untreated controls, and EGCG withdrawal for 7 days resulted in reductions of $33 \%$ in total p53, 52\% in Lys373 and 39\% in Lys 382 in the treated cells. (Fig. 3A and B).

GTPs and EGCG cause increased expression of p21/wafl and Bax in human prostate cancer cells. We then studied the levels of p21/waf1 and Bax in response to GTP and EGCG exposure. The elevation of p53 levels by GTPs and EGCG resulted in a significant increase in $\mathrm{p} 21 / \mathrm{waf} 1$ and Bax expression both at the protein and message levels. As shown in Fig. 4A and B, exposure of cancer cells to $2.5-10 \mu \mathrm{g} / \mathrm{ml}$ doses of GTPs resulted in increased p21/waf1 protein levels, ranging from $80-120 \%$ at 3 days to $123-151 \%$ at 7 days, and increased Bax protein levels, ranging from $110-142 \%$ at 3 days to $138-164 \%$ at 7 days, 
A

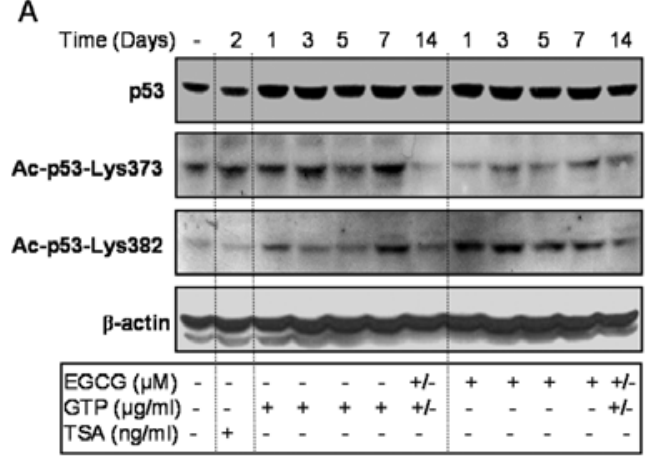

B

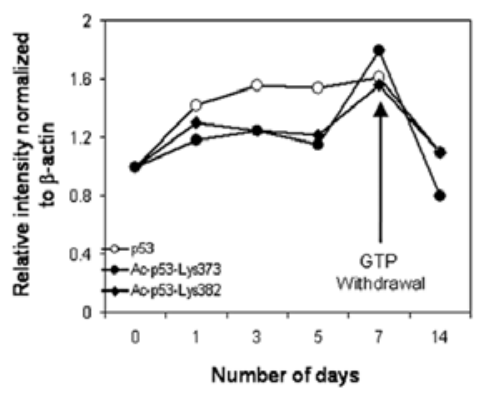

C

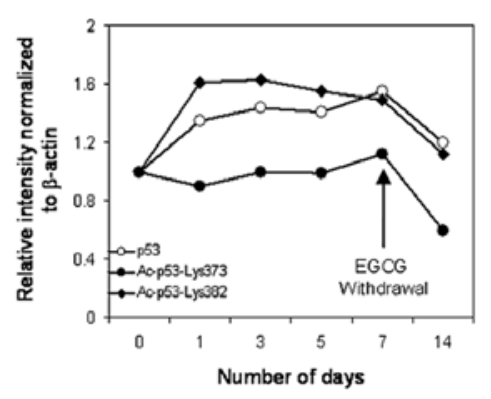

Figure 3. Reversal of the effect of green tea polyphenols (GTPs) and (-) epigallocatechin-3-gallate (EGCG) on the p53 protein and its acetylation on C-terminal Lys373 and Lys382 in LNCaP human prostate cancer cells after removal of GTPs and EGCG. (A) Cells were treated with GTPs (5 $\mu \mathrm{g} / \mathrm{ml})$ and EGCG (20 $\mu \mathrm{M})$ and the levels of p53 protein and its acetylation on C-terminal Lys373 and Lys382 were monitored in a time-dependent manner for up to 7 days. After treatment, GTPs or EGCG were removed from the medium for another 7 days and the levels of p53 protein as well as its acetylation on C-terminal Lys 373 and Lys 382 were monitored. (B) Relative intensities of p53 protein and its acetylation on C-terminal Lys 373 and Lys 382 normalized to $\beta$-actin plotted in the form of a line graph in LNCaP cells. The details are provided in Materials and methods.

compared to the levels in untreated cells. Similarly, EGCG treatment at the doses of 5-20 $\mu \mathrm{M}$ caused an increase in $\mathrm{p} 21 / \mathrm{waf} 1$ protein levels which ranged from $110-133 \%$ at 3 days to $128-148 \%$ at 7 days, and increased Bax levels ranging from $134-154 \%$ at 3 days to $148-166 \%$ after 7 days of EGCG exposure. Treatment of cells with TSA resulted in a $124 \%$ increase in $\mathrm{p} 21 /$ waf 1 and a $156 \%$ increase in Bax, compared to the untreated cells (Fig. 4A and B).

GTP and EGCG treatments resulted in significant increases in mRNA expression of $\mathrm{p} 21 / \mathrm{waf} 1$ and Bax in LNCaP prostate cancer cells. A 121-138\% increase in the p21/waf1 and a $118-123 \%$ increase in the Bax message level were observed after 3 days of GTP exposure, and a $119-123 \%$ increase in the p21/waf1 and a 128-140\% increase in the Bax message level were observed after 3 days of EGCG exposure, compared to the untreated cells. Similar responses in p21/waf1 and Bax were noted after TSA exposure: a 112\% increase in p21/waf1 and a $145 \%$ increase in Bax message levels were noted after 2 days of $20 \mathrm{ng} / \mathrm{ml}$ treatment (Fig. 4C and D).

GTPS and EGCG cause increased binding of acetylated p53-Lys373 to the promoters of p21/wafl and Bax genes. We then investigated changes in the acetylation status of p53 at Lys373 with the promoter region of the p21/wafl and Bax genes. Using anti-acetylated p53-Lys373 antibody followed by PCR with the primers specific for p21/wafl and Bax promoters, ChIP assay was performed. As shown in Fig. 5, GTP treatment resulted in a dose-dependent increase in the amount of acetylated p53-Lys373 associated with the p21/ waf1 and Bax promoter. GTP exposure at 5 and $10 \mu \mathrm{g} / \mathrm{ml}$ resulted in a $155-164 \%$ increase in the expression of $\mathrm{p} 21 / \mathrm{waf} 1$ and a $129-135 \%$ increase in Bax expression in LNCaP cells compared to the untreated controls. Similar results were noted with EGCG exposure at a dose of $10 \mu \mathrm{M}$, which caused a $148 \%$ increase in p21/waf1 and a 141\% increase in Bax expression, compared to the untreated cells (Fig. 5A and B).

GTPS and EGCG cause cell cycle arrest and induce apoptosis in human prostate cancer cells. We then examined the effect of GTPs and EGCG on the inhibition of cell growth and induction of apoptosis in $\mathrm{LNCaP}$ human prostate cancer cells. GTP $(2.5-10 \mu \mathrm{g} / \mathrm{ml})$ and EGCG $(5-20 \mu \mathrm{M})$ treatment at these low doses caused a modest reduction of $4-9 \%$ in cell density after 7 days of exposure (data not shown). Analysis of DNA content by flow cytometry showed that GTPs caused a marked increase in the percentage of cells in the G0-G1-phase of the cell cycle. Compared with the vehicle-treated controls, GTP treatment resulted in an appreciable arrest of LNCaP cells in the G0/G1 phase of cell cycle after 3 days of the treatment. The treatment caused an arrest of $60.3 \%$ of cells in the G0/G1 phase of the cell cycle at $5 \mu \mathrm{g} / \mathrm{ml}$ that further increased to $72.2 \%$ at $10 \mu \mathrm{g} / \mathrm{ml}$, compared to the controls (46.9\%). Similar observations were noted following EGCG treatment of cells at 10 and $20 \mu \mathrm{M}$ doses, which resulted in 61.2 and $74.4 \%$ arrest compared to $45.8 \%$ in the vehicle-treated controls, respectively. Furthermore, exposure of LNCaP cells to $20 \mathrm{ng} / \mathrm{ml}$ TSA for 2 days caused an increase of $84.8 \%$ of cells in the G0/G1 phase compared to $59.4 \%$ in the untreated group. This increase in the G0/G1 cell population was accompanied by a concomitant decrease of cell numbers in the S-phase and G2-M-phase of the cell cycle (Fig. 6A). Furthermore, the percentages of cells in G0/G1 phase arrest and cells undergoing cell death were determined by measuring the sub-G1 cell population. Exposure of LNCaP cells to GTPs $(5-10 \mu \mathrm{g} / \mathrm{ml})$ for 7 days resulted in significantly increased cell death, ranging from $10.7-17.4 \%$, compared to $2.2 \%$ cell death in the control populations. EGCG exposure $(10-20 \mu \mathrm{M})$ also resulted in increased cell death: $10.3-18.2 \%$, compared to $2.7 \%$ in the control cells. Exposure of cells to $20 \mathrm{ng} / \mathrm{ml}$ TSA for 2 days resulted in a modest increase in cell death $(4.9 \%)$, compared to $1.36 \%$ in the untreated group (Fig. 6B). These observations positively correlate with increases in p21/waf1 and Bax levels in LNCaP cells after GTP and EGCG treatment.

\section{Discussion}

Class I HDACs are overexpressed in the majority of human cancers, including prostate cancer (10-12). The inhibition of 
A

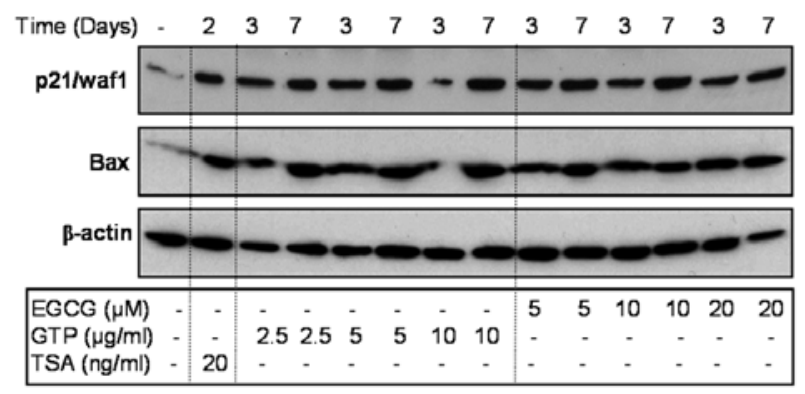

B

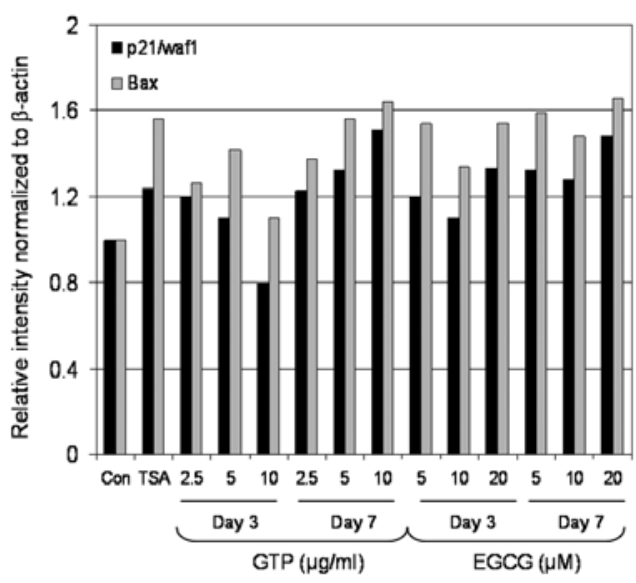

D

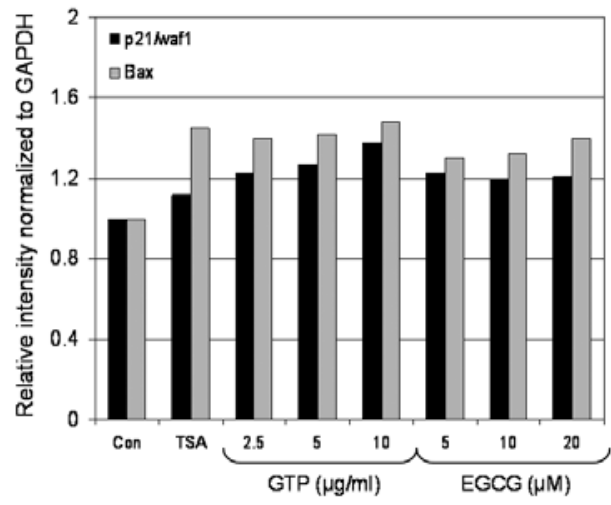

Figure 4. Effect of green tea polyphenols (GTP) and its major constituent (-) epigallocatechin-3-gallate (EGCG) and HDAC inhibitor, trichostatin A (TSA), on the expression of p21/waf1 and Bax at transcriptional and message levels in LNCaP human prostate cancer cells. (A) Cells were treated with GTPs (2.5-10 $\mu \mathrm{g} / \mathrm{ml})$ or EGCG $(5-20 \mu \mathrm{M})$ for 3 and 7 days or TSA $(20 \mathrm{ng} / \mathrm{ml})$ for 2 days and western blotting was performed for p21/waf1 and Bax levels in LNCaP cells. (B) Relative intensities of p21/waf1 and Bax proteins normalized to $\beta$-actin in LNCaP cells. (C) Estimation of mRNA levels of p21/waf1 and Bax in LNCaP cells as determined by RT-PCR. (D) Relative intensities of p21 and Bax mRNA levels normalized to GAPDH in LNCaP cells. The details are provided in Materials and methods.

A

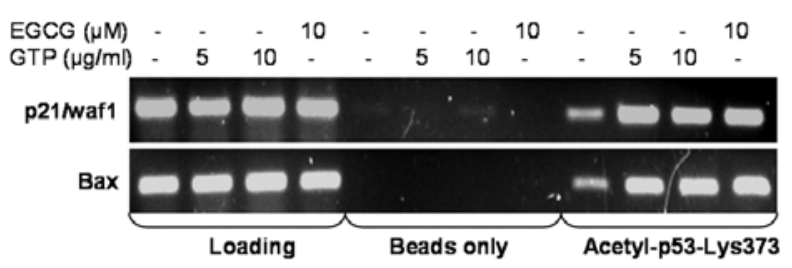

B

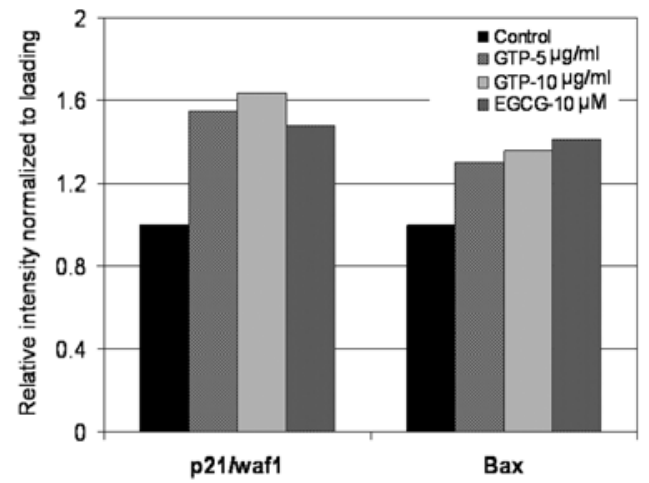

Figure 5. Effect of green tea polyphenols (GTPs) and their major constituent (-) epigallocatechin-3-gallate (EGCG) in the binding of acetylated p53-Lys373 to the promoters of $\mathrm{p} 21 / \mathrm{wafl}$ and Bax genes. Chromatin immunoprecipitation (ChIP) assay was performed for association of acetylated p53-Lys373 with the promoters of $\mathrm{p} 21 / \mathrm{waf} 1$ and Bax in LNCaP cells. (A) Treatment of LNCaP cells with GTPs (5-10 $\mu \mathrm{g} / \mathrm{ml})$ and EGCG $(10 \mu \mathrm{M})$ caused an increased association of acetylated p53-Lys373 with the promoters of p21/waf1 and Bax. (B) Relative intensities of amplified promoters of $p 21 /$ wafl and Bax genes normalized to loading. The details are provided in Materials and methods.

their activity by HDAC inhibitors, such as SAHA and TSA in neoplastic cells in culture induces differentiation and/or apoptosis $(13,14)$. HDAC inhibitors have also been reported to inhibit tumor growth in in vivo studies $(15,16)$. In this study, 
A

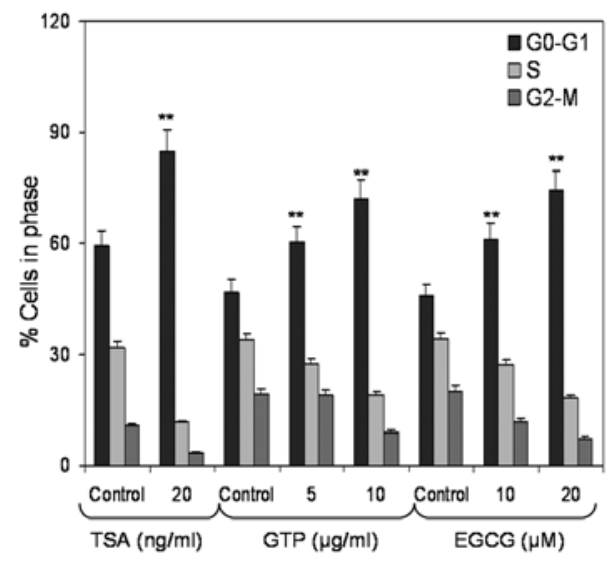

B

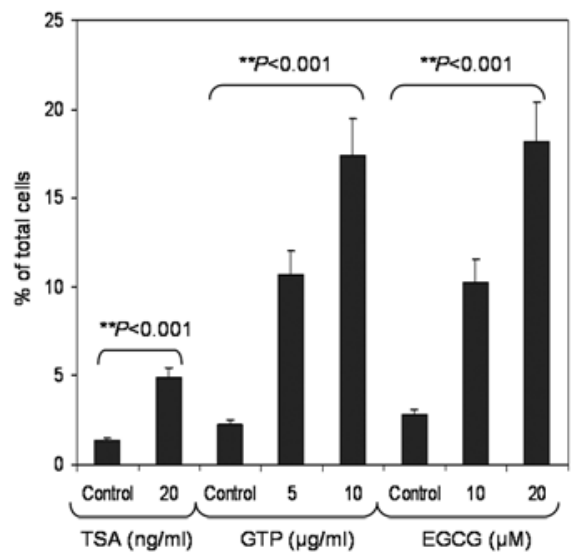

Figure 6. Effect of green tea polyphenols (GTPs) and their major constituent (-) epigallocatechin-3-gallate (EGCG) and the HDAC inhibitor, trichostatin A (TSA), on cell cycle arrest and induction of apoptosis in LNCaP human prostate cancer cells. (A) Cells were treated with GTPs (5-10 $\mu$ g/ml) and EGCG (10-20 $\mu \mathrm{M})$ for 3 days and TSA (20 ng/ml) for 2 days and the distribution of cells was recorded at different stages of the cell cycle using FACS analysis. (B) LNCaP cells were treated with GTPs $(5-10 \mu \mathrm{g} / \mathrm{ml})$ and EGCG $(10-20 \mu \mathrm{M})$ for 7 days and TSA (20 ng/ml) for 2 days and the number of cells undergoing apoptosis was determined by measuring the cell population in the sub-G1 phase of the cell cycle. The details are provided in Materials and methods.

we show that GTPs inhibit class I HDACs in prostate cancer cells, thereby altering the dynamic balance between acetylation/deacetylation and resulting in increased acetylation of p53 at its C-terminus at Lys373 and Lys382. We also show that GTPs enhance p53 stabilization and DNA binding to the promoters of p21/wafl and Bax genes, leading to cell cycle arrest and apoptosis of LNCaP prostate cancer cells. Previous investigators have reported that prostate cancer cells harboring wild-type p53 undergo cell cycle arrest and apoptosis after treatment with GTPs and EGCG, effects associated with activation of p53 and its downstream targets $(17,18)$, although the exact mechanism of p53 activation is not well understood. To our knowledge, the present study is the first to demonstrate that GTPs and EGCG have the ability to stabilize p53 by increasing its acetylation as a function of inhibition of class I HDACs.

In our previous study, we demonstrated the stabilization of the acetylated histones by GTPs at physiologically attainable concentrations as early as $24 \mathrm{~h}$ of treatment, well before the morphological evidence of apoptosis and cell cycle arrest (9), suggesting that the re-expression of genes that regulate differentiation or cell death through changes in chromatin conformation might be responsible for the effects of GTPs on cell cycle kinetics and apoptosis. In addition to stabilizing the acetylation of histones, treatment with GTPs and EGCG also causes an increase in the levels of acetylated p53 and total p53 levels in LNCaP cells. Stabilization of p53 leads to the activation of downstream targets including p21/waf1 and Bax due to the p53 binding sites in the promoter of these genes. Consistent with this idea, we have shown that the stabilization of acetylated Lys373 p53 by GTPs and EGCG induces the upregulation of $\mathrm{p} 21 / \mathrm{waf} 1$ and Bax protein levels in prostate cancer LNCaP cells.

Previous reports have demonstrated that the acetylation of p53 is important in suppressing oncogenic Ras-induced transformation and inducing metaphase chromosome fragility, suggesting that acetylation may be essential for the tumor suppressor function of p53 (19,20). The findings that p300/ CBP acetyltranferase and p19/ARF promote p53 acetylation in vivo, while MDM2 inhibits acetylation, further support the concept that acetylation is an important modification targeted by both positive and negative regulators critical for p53 tumorsuppressor activity (21-23). Our study demonstrates that GTPs and EGCG have the ability to acetylate p53 at Lys373 and Lys382, leading to increased levels of total p53 in LNCaP cells.

Certain proteins such as MDM2, a negative regulator of p53, possess an E3-like ubiquitin ligase activity which rapidly promotes the degradation of p53 so as to retain p53 at a low level in unstressed cells (24). Studies have shown that the Lys320 site at p53 is acetylated in LNCaP prostate cancer cells and remains unaffected by treatment with HDAC inhibitors (5). Acetylation of p53 at either Lys373 or Lys382 blocks the MDM2-mediated ubiquitination of p53, which increases the steady state level of acetylated proteins (25-27). Our results show that GTPs and EGCG cause an increase in acetylation at both Lys373 and Lys382 of p53 but not at Lys320, which leads to p53-dependent transcriptional activation. Previously, we demonstrated that EGCG-mediated phosphorylation of p53 also results in the reduced interaction of p53 with its negative regulator, MDM2, in human prostate cancer cells. EGCG exposure also results in increased expression of p14ARF, a tumor suppressor that negatively regulates MDM2 (28). Our results indicate that GTPs and EGCG stabilize p53 via both phosphorylation and acetylation through modulation of the ARF-MDM2 pathway, as well as through inhibition of class I HDACs.

Previous studies have demonstrated that p53 cooperates with the transcription factor Sp1, through binding to the C-terminal domain of Sp1, to induce transcription of the p21 gene, whereas the binding of HDAC1 to the same domain inhibits this transcription (29). Our study demonstrates that GTPs and EGCG have the ability to inhibit HDAC1 after 3 days of exposure, which may contribute to the induction of p21 transcription via p53 activation in LNCaP cells. 
Furthermore, GTPs cause p21/waf1 activation in p53 null PC-3 human prostate cancer cells, through a p53-independent mechanism. In addition, the ability of the different acetylated isoforms to recruit distinct co-activator complexes to the p21/ waf1 promoter is another contributing factor in determining the downstream events associated with HDAC inhibitors. For example, Ac-Lys373 p53 recruits p300, forkhead transcription factors, the androgen receptor and Brg-1. When assembled on the 553 response elements in the $\mathrm{p} 21 /$ waf1 promoter, this complex induces the assembly of TBP, RNA Pol II, and TFIIH on the basal promoter and initiates p21/waf1 transcription. In contrast, Ac-Lys 382 p53 recruits CBP and Brm- 1 to the p53 response elements in the proximal $\mathrm{p} 21 / \mathrm{waf} 1$ promoter, but this complex does not recruit TBP, RNA Pol II, or TFIIH to the basal promoter, suggesting that Ac-Lys382-p53 promotes the assembly of a non-functional complex on the p21/waf1 promoter (30). Additional studies of the recruitment of various co-activator complex after exposure of $\mathrm{p} 53^{+/+}$and $\mathrm{p} 53^{-/-}$cells with GTPs and EGCG in the regulation of $\mathrm{p} 21 /$ waf1 may prove fruitful.

Members of the class I HDAC family (HDACs 1, 2, 3 and 8) are localized in the nucleus of cells and are ubiquitously expressed. Class I HDACs are overexpressed in many human cancers including prostate cancer (10-12) HDAC inhibitors promote increased acetylation of histone and non-histone proteins, including p53. However, different class I HDACs may regulate $\mathrm{p} 53$ activity in different ways, which may or may not involve p53 acetylation. For example, deacetylation of p53 by HDAC1 and HDAC2 results in the degradation of deacetylated p53; HDAC inhibitors have been shown to reverse this process (31). The inhibition of HDAC1 in cancer cells results in the acetylation of the DNA binding domain of p53 and causes apoptosis (32). The loss of HDAC1 may be compensated by the upregulation of HDAC2 and HDAC3 (33). HDAC2 has been shown to inhibit $\mathrm{p} 53$ binding onto its promoter, and inhibition of HDAC2 increases p53 binding without effecting its acetylation (34). These studies indicate that each member of the class I HDAC family may have a different mechanism of action, and the findings furthermore imply that the binding of p 53 to DNA is a critical mechanism for regulation of its activity, which may or may not require HDAC enzymatic activity to modify p53 acetylation. Our study demonstrates that GTPs and EGCG have the ability to suppress HDAC activity and expression. Further studies are required to elucidate their effects on individual HDACs in regulating p53 function.

Previous studies have shown that HDACs, in particular class I HDACs, form complexes with p53 and significantly deacetylate $\mathrm{p} 53$, reducing its ability to bind to Bax. By contrast, HDAC inhibitors have been shown to induce the acetylation of p53 and trigger the transactivation of pro-apoptotic genes such as Bax, PUMA, Noxa, and the transcriptional repressor of the anti-apoptotic proteins, Bcl2 and Mcl-1 (7,35-37). In our study, GTPs and EGCG stimulated Bax induction at the transcriptional and message levels in $\mathrm{LNCaP}$ cells, as a consequence of enhanced binding of Ac-p53-Lys373 to the Bax promoter. In addition to the roles played by 553 as a transcription factor, Ac-p53 also promotes cell death independent of transcription by disrupting the binding of Mcl-1 to Bak or by destroying the Ku-Bax complex (38). Further studies are required in order to investigate possible p53-independent functions of GTPs and
EGCG in the induction of Bax in prostate cancer cells with various levels of $\mathrm{p} 53$ expression.

Our study confirms that class I HDACs participate in p53-mediated gene regulation in human prostate cancer cells, and provides further insight into the mechanisms whereby GTPs alter p53-mediated gene regulation of cell cycle arrest and apoptosis. Perhaps further studies on the potential beneficial effects of GTPs may lead to the development of novel, safe and effective preventive and/or therapeutic strategies in the management of prostate cancer patients.

\section{Acknowledgements}

This study was supported by the United States Public Health Service Grants, RO1 CA115491 and R21 CA109424, to S.G.

\section{References}

1. Bai L and Zhu WG: p53: structure, function and therapeutic applications. J Cancer Mol 2: 141-153, 2006.

2. Tang Y, Zhao W, Chen Y, Zhao Y and Gu W: Acetylation is indispensable for p53 activation. Cell 133: 612-626, 2008.

3. Yang XJ and Seto E: HATs and HDACs: from structure, function and regulation to novel strategies for therapy and prevention. Oncogene 26: 5310-5318, 2007.

4. Weichert W: HDAC expression and clinical prognosis in human malignancies. Cancer Lett 280: 168-176, 2009.

5. Roy S, Packman K, Jeffrey R and Tenniswood M: Histone deacetylase inhibitors differentially stabilize acetylated p53 and induce cell cycle arrest or apoptosis in prostate cancer cells. Cell Death Differ 12: 482-491, 2005.

6. Condorelli F, Gnemmi I, Vallario A, Genazzani AA and Canonico PL: Inhibitors of histone deacetylase (HDAC) restore the p53 pathway in neuroblastoma cells. Br J Pharmacol 153: 657-668, 2008.

7. Luo J, Li M, Tang Y, Laszkowska M, Roeder RG and Gu W: Acetylation of p53 augments its site-specific DNA binding both in vitro and in vivo. Proc Natl Acad Sci USA 101: 2259-2264, 2004.

8. Thakur VS, Gupta K and Gupta S: Green tea polyphenols causes cell cycle arrest and apoptosis in prostate cancer cells by suppressing class I histone deacetylases. Carcinogenesis 33: 377-384, 2012.

9. Pandey M, Shukla S and Gupta S: Promoter demethylation and chromatin remodeling by green tea polyphenols leads to re-expression of GSTP1 in human prostate cancer cells. Int J Cancer 126: 2520-2533, 2010.

10. Nakagawa M, Oda Y,Eguchi T, Aishima S, Yao T,Hosoi F, Basaki Y, Ono M, Kuwano M, Tanaka M and Tsuneyoshi M: Expression profile of class I histone deacetylases in human cancer tissues. Oncol Rep 18: 769-774, 2007.

11. Weichert W, Röske A, Gekeler V, Beckers T, Stephan C, Jung K, Fritzsche FR, Niesporek S, Denkert C, Dietel M and Kristiansen G: Histone deacetylases 1,2 and 3 are highly expressed in prostate cancer and HDAC2 expression is associated with shorter PSA relapse time after radical prostatectomy. Br J Cancer 98: 604-610, 2008.

12. FritzscheFR, Weichert W, Röske A, Gekeler V,Beckers T, Stephan C, Jung K, Scholman K, Denkert C, Dietel M and Kristiansen G: Class I histone deacetylases 1,2 and 3 are highly expressed in renal cell cancer. BMC Cancer 8: 381, 2008.

13. Kim YB, Ki SW, Yoshida M and Horinouchi S: Mechanism of cell cycle arrest caused by histone deacetylase inhibitors in human carcinoma cells. J Antibiot (Tokyo) 53: 1191-1200, 2000.

14. Mai A, Massa S, Rotili D, Cerbara I, Valente S, Pezzi R, Simeoni S and Ragno R: Histone deacetylation in epigenetics: an attractive target for anticancer therapy. Med Res Rev 25: 261-309, 2005.

15. Vigushin DM, Ali S, Pace PE, Mirsaidi N, Ito K, Adcock I and Coombes RC: Trichostatin A is a histone deacetylase inhibitor with potent antitumor activity against breast cancer in vivo. Clin Cancer Res 7: 971-976, 2001.

16. Cohen LA, Amin S, Marks PA, Rifkind RA, Desai D and Richon VM: Chemoprevention of carcinogen-induced mammary tumorigenesis by the hybrid polar cytodifferentiation agent, syberanilohydroxamic acid (SAHA). Anticancer Res 19: 4999-5005, 1999. 
17. Hastak K, Agarwal MK, Mukhtar H and Agarwal ML: Ablation of either p21 or Bax prevents p53 dependent apoptosis induced by green tea polyphenol epigallocatechin-3-gallate. FASEB J 19: 789-791, 2005

18. Thakur VS, Ruhul Amin AR, Paul RK, Gupta K, Hastak K, Agarwal MK, Jackson MW, Wald DN, Mukhtar H and Agarwal ML: p53-Dependent p21-mediated growth arrest pre-empts and protects HCT116 cells from PUMA-mediated apoptosis induced by EGCG. Cancer Lett 296: 225-232, 2010

19. Pearson M, Carbone R, Sebastiani C, Cioce M, Fagioli M, Saito S, Higashimoto Y, Appella E, Minucci S, Pandolfi PP and Pelicci PG: PML regulates p53 acetylation and premature senescence induced by oncogenic Ras. Nature 406: 207-210, 2000.

20. Yu A, Fan HY, Liao D, Bailey AD and Weiner AM: Activation of $\mathrm{p} 53$ or loss of the Cockayne syndrome group $\mathrm{B}$ repair protein causes metaphase fragility of human U1, U2, and 5S genes. Mol Cell 5: 801-810, 2000

21. Liu L, Scolnick DM, Trievel RC, Zhang HB, Marmorstein R, Halazonetis TD and Berger SL: p53 sites acetylated in vitro by PCAF and $\mathrm{p} 300$ are acetylated in response to DNA damage. Mol Cell Biol 19: 1202-1209, 1999.

22. Zhang Y, Xiong Y and Yarbrough WG: ARF promotes MDM2 degradation and stabilizes p53: ARF INK4a locus deletion impairs both the $\mathrm{Rb}$ and p53 tumor suppression pathways. Cell 92: 725-734, 1998.

23. Ito A, Lai CH, Zhao X, Saito S, Hamilton MH, Appella E and Yao TP: p300/CBP-mediated p53 acetylation is commonly induced by $\mathrm{p} 53$-activating agents and inhibited by MDM2 EMBO J 20: 1331-1340, 2001.

24. Haupt Y, Maya R, Kazaz A and Oren M: Mdm2 promotes the rapid degradation of p53. Nature 387: 296-299, 1997.

25. Li M, Luo J, Brooks CL and Gu W: Acetylation of p53 inhibits its ubiquitination by Mdm2. J Biol Chem 277: 50607-50611, 2002.

26. Ito A, Kawaguchi Y, Lai CH, Kovacs JJ, Higashimoto Y, Appella E and Yao TP: MDM2-HDAC1-mediated deacetylation of p53 is required for its degradation. EMBO J 21: 6236-6345, 2002.

27. Zhao Y, Lu S, Wu L, Chai G, Wang H, Chen Y, Sun J, Yu Y, Zhou W, Zheng Q, Wu M, Otterson GA and Zhu WG: Acetylation of $\mathrm{p} 53$ at lysine $373 / 382$ by the histone deacetylase inhibitor depsipeptide induces expression of p21(Waf1/Cip1). Mol Cell Biol 26: 2782-2790, 2006.

28. Hastak K, Gupta S, Ahmad N, Agarwal MK, Agarwal ML and Mukhtar $\mathrm{H}$ : Role of $\mathrm{p} 53$ and $\mathrm{NF}-\kappa \mathrm{B}$ in epigallocatechin3 -gallate-induced apoptosis of LNCaP cells. Oncogene 22: 4851-4859, 2003.
29. Lagger G, Doetzlhofer A, Schuettengruber B, Haidweger E, Simboeck E, Tischler J, Chiocca S, Suske G, Rotheneder H, Wintersberger E and Seiser C: The tumor suppressor p53 and histone deacetylase 1 are antagonistic regulators of the cyclindependent kinase inhibitor p21/WAF1/CIP1 gene. Mol Cell Biol 23: 2669-2679, 2003.

30. Roy S and Tenniswood M: Site-specific acetylation of p53 directs selective transcription complex assembly. J Biol Chem 282: 4765-4771, 2007.

31. Wilting RH, Yanover E, Heideman MR, Jacobs H, Horner J, van der Torre J, DePinho RA and Dannenberg JH: Overlapping functions of Hdac1 and Hdac2 in cell cycle regulation and hematopoiesis. EMBO J 29: 2586-2597, 2010.

32. Juan LJ, Shia WJ, Chen MH, Yang WM, Seto E, Lin YS and Wu CW: Histone deacetylases specifically down-regulate p53-dependent gene activation. J Biol Chem 275: 20436-20443, 2000

33. Montgomery RL, Davis CA, Potthoff MJ, Haberland M, Fielitz J, Qi X, Hill JA, Richardson JA and Olson EN: Histone deacetylases 1 and 2 redundantly regulate cardiac morphogenesis, growth, and contractility. Genes Dev 21: 1790-1802, 2007.

34. Harms KL and Chen X: Histone deacetylase 2 modulates p53 transcriptional activities through regulation of p53-DNA binding activity. Cancer Res 67: 3145-3152, 2007.

35. Levine AJ: p53, the cellular gatekeeper for growth and division. Cell 88: 323-331, 1997.

36. Terui T, Murakami K, Takimoto R, Takahashi M, Takada K, Murakami T, Minami S, Matsunaga T, Takayama T, Kato J and Niitsu Y: Induction of PIG3 and NOXA through acetylation of p53 at 320 and 373 lysine residues as a mechanism for apoptotic cell death by histone deacetylase inhibitors. Cancer Res 63: 8948-8954, 2003.

37. Pietrzak M and Puzianowska-Kuznicka M: p53-dependent repression of the human MCL-1 gene encoding an anti-apoptotic member of the BCL-2 family: the role of Spl and of basic transcription factor binding sites in the MCL-1 promoter. Biol Chem 389: 383-393, 2008.

38. Yamaguchi H, Woods NT, Piluso LG, Lee HH, Chen J, Bhalla KN, Monteiro A, Liu X, Hung MC and Wang HG: p53 acetylation is crucial for its transcription-independent proapoptotic functions. J Biol Chem 284: 11171-11183, 2009. 